\title{
Regional immunity in melanoma: immunosuppressive changes precede nodal metastasis
}

\author{
Aaron S Mansfield ${ }^{1,2}$, Shernan G Holtan ${ }^{1,2}$, Travis E Grotz ${ }^{3}$, Jake B Allred ${ }^{4}$, James W Jakub ${ }^{3}$, \\ Lori A Erickson ${ }^{5}$ and Svetomir N Markovic ${ }^{1,2}$ \\ ${ }^{1}$ Division of Hematology, Department of Internal Medicine, Mayo Clinic, Rochester, MN, USA; ${ }^{2}$ Department of \\ Oncology, Mayo Clinic, Rochester, MN, USA; ${ }^{3}$ Department of Surgery, Mayo Clinic, Rochester, MN, USA; \\ ${ }^{4}$ Cancer Center Statistics, Mayo Clinic, Rochester, MN, USA and ${ }^{5}$ Department of Laboratory Medicine and \\ Pathology, Mayo Clinic, Rochester, MN, USA
}

\begin{abstract}
In order to characterize the degree of immunosuppression in regional immunity in patients with melanoma, we used immunohistochemistry to analyze markers of T-cell subtype and polarity, costimulation, dendritic cell maturation, monocytes, lymphatic vasculature, and angiogenesis. Specifically, we analyzed expression of CD4, CD8, CD14, CD40, CD86, CD123, HLA-DR, IL-10, LYVE, VEGFR3, and VEGF-C in lymph nodes. We compared sentinel lymph nodes with and without metastasis from patients with melanoma with both infection inflamed (reactive) and dormant human lymph nodes. There were no differences demonstrated between sentinel lymph nodes with or without metastasis from patients with melanoma in any of the markers that were tested. Both groups of sentinel lymph nodes had fewer $\mathrm{CDB}^{+} \mathrm{T}$ cells than either set of control nodes. Whereas the infection inflamed lymph nodes demonstrated Th2 polarity, the dormant lymph nodes demonstrated Th1 polarity. In conclusion, changes in regional immunity appeared to precede metastasis in melanoma. Whether there was tumor present in sentinel lymph nodes or not, these nodes demonstrated a marked decrease in cytotoxic $T$ cells compared with both sets of controls. Furthermore, the control lymph nodes used for comparison can significantly impact interpretation, as the dormant and reactive lymph nodes markedly varied in their immune profiles. These immunologic changes may explain the successful metastasis of melanoma in the midst of the immune environment of the sentinel lymph node, and lend insights into the mechanisms of lymphatic metastases in other solid malignancies.
\end{abstract}

Modern Pathology (2011) 24, 487-494; doi:10.1038/modpathol.2010.227; published online 10 December 2010

Keywords: angiogenesis; cytotoxic T cells; lymph nodes; melanoma; regional immunity

Lymph node metastasis is one of the most important prognostic factors in melanoma. ${ }^{1}$ Whereas patients without nodal involvement generally have excellent outcomes, those with nodal involvement more commonly experience recurrence after resection and diminished survival. Previous investigations suggest that melanoma-draining lymph nodes demonstrate immunologic alterations and are likely immunosuppressed. ${ }^{2}$ Sentinel lymph nodes are the first lymph nodes to drain a tumor bed, and represent the first expected site of metastasis of

Correspondence: Dr SN Markovic, MD, PhD, Mayo Clinic 200 First Street SW, Rochester, MN 55905, USA.

E-mail: markovic.svetomir@mayo.edu

Received 17 July 2010; revised 30 August 2010; accepted 2

September 2010; published online 10 December 2010 melanoma. ${ }^{3,4}$ Sentinel lymph nodes are a site of contact between tumor-associated antigens and the adaptive immune system and represent a regional unit of metastasis and immune response. Accordingly, sentinel lymph nodes are an ideal setting for the investigations of the early immunologic (endocrine and paracrine) events that lead up to melanoma progression from a primary tumor to metastatic cancer. In the past year, we have described that patients with metastatic melanoma exist in a state of systemic chronic inflammation driven by tumorderived vascular endothelial growth factor (VEGF) overproduction leading to systemic immune polarization to a state of CD4 cell Th2 bias. ${ }^{5}$ Consequently, we sought to determine if similar differences in regional immune profiles in sentinel lymph nodes of patients with melanoma existed. 
Presented is our immunohistochemical analysis of sentinel lymph nodes with and without evidence of melanoma metastases compared with infection inflamed and dormant human lymph nodes.

\section{Materials and methods}

\section{Patients}

Archival formalin-fixed paraffin-embedded sentinel lymph nodes from patients with melanoma with (SLN,$+ n=8$ ), or without (SLN-, $n=12$ ) detectable sentinel lymph nodes metastases were used for the analysis (Table 1). Control infection inflamed 'reactive' lymph nodes $(n=10)$ and dormant lymph nodes $(n=10)$ were also collected and analyzed. Reactive lymph nodes were from patients with no evidence of malignancy who presented with lymphadenopathy and underwent lymph node excision for diagnosis, or underwent lymph node excision in conjunction with another primary operation. Dormant lymph nodes were collected from patients with breast cancer who underwent prophylactic contralateral mastectomy in conjunction with sentinel lymph node biopsy. These patients had no known active cancer or inflammatory process at the time of lymph node excision. This retrospective study was reviewed and approved by the Mayo Clinic Institutional Review Board.

\section{Immunohistochemistry}

Formalin-fixed paraffin-embedded nodes were sectioned into 3 to $4 \mu \mathrm{m}$ slices and affixed on glass slides. Samples were heated for half an hour at $56{ }^{\circ} \mathrm{C}$, and then deparaffinized in xylene, rehydrated in a graded alcohol series, and washed in water. Heat-induced epitope retrieval was used for antigen retrieval. Endogenous peroxidase activity was quenched in a bath of methanol and hydrogen peroxide. Only one stain was used per slide. We stained samples for CD4 (no. ab864; Abcam Cambridge, MA, USA) at 1:100, CD8 (no. ab4055; Abcam) at 1:100, CD14 (no. ab8676; Abcam) at 1:50, CD40 (no. ab27224; Abcam) neat, CD86 (no. AF-141-MA; R\&D Systems, Minneapolis, MN, USA) at 1:20, CD123 (no. 14-1234; eBiosciences, San Diego, CA, USA) at 1:100, HLA-DR (no. 14-990-82; eBiosciences) at 1:100, LYVE (no. DP3500; Acris, Herford, Germany) at 1:25, IL-10 (no. ab33471; Abcam) at 1:400, VEGFR3 (cat no. ab27278; Abcam) at 1:100, VEGF-C (no. sc1881; Santa Cruz Biotechnology, Santa Cruz, CA, USA) at 1:100, Foxp3 (ab22510; Abcam) at 1:100, T-bet (no. MAB5385; R\&D) at 1:50, GATA-3 (BAF2605; R\&D) at 1:5, Galectin 3 (no. ab58086; Abcam) at 1:200, placental growth factor (no. ab9542; Abcam) at 1:1000, osteopontin (MAB14331; R\&D) at 1:10, and HLA-G (no. ab7759; Abcam) at 1:100 (the reported function of each marker is described in Table 2). Samples

Table 1 Patient characteristics

\begin{tabular}{|c|c|c|c|c|}
\hline & $\begin{array}{l}\text { Sentinel lymph nodes } \\
\text { with metastasis }\end{array}$ & $\begin{array}{l}\text { Sentinel lymph nodes } \\
\text { without metastasis }\end{array}$ & $\begin{array}{c}\text { Dormant } \\
\text { lymph nodes }\end{array}$ & $\begin{array}{c}\text { Reactive } \\
\text { lymph nodes }\end{array}$ \\
\hline Number & 8 & 12 & 10 & 10 \\
\hline Age (years) ${ }^{\mathrm{a}}$ & $63(55-74)$ & $43(36-67)$ & $52(49-67)$ & $50(42-59)$ \\
\hline \multicolumn{5}{|l|}{ Sex } \\
\hline Male & 5 & 4 & 0 & 2 \\
\hline Female & 3 & 8 & 10 & 8 \\
\hline Tumor thickness $(\mathrm{mm})^{\mathrm{a}}$ & $3.5(1.9-5.2)$ & $1.8(1.1-2.2)$ & & \\
\hline \multicolumn{5}{|l|}{ Tumor stage $\mathrm{e}^{\mathrm{b}}$} \\
\hline Ia & & 2 & & \\
\hline Ib & & 5 & & \\
\hline IIa & & 1 & & \\
\hline IIb & & 4 & & \\
\hline IIIa & 4 & & & \\
\hline IIIb & 2 & & & \\
\hline IIIc & 2 & & & \\
\hline \multicolumn{5}{|l|}{ Location } \\
\hline Axillary & $4(50 \%)$ & $6(50 \%)$ & $10(100 \%)$ & $1(10 \%)$ \\
\hline Inguinal & $4(50 \%)$ & $6(50 \%)$ & & \\
\hline Cervical & & & & $2(20 \%)$ \\
\hline Mediastinal & & & & $2(20 \%)$ \\
\hline Mesenteric & & & & $1(10 \%)$ \\
\hline Pericystic (gall bladder) & & & & $2(20 \%)$ \\
\hline Perinephric & & & & $1(10 \%)$ \\
\hline Periparotid & & & & $1(10 \%)$ \\
\hline
\end{tabular}

${ }^{\mathrm{a}}$ Median, 25-75\% quartile.

${ }^{\mathrm{b}}$ As defined by the American Joint Committee of Melanoma Staging Cancer System. ${ }^{48}$ 
Table 2 Summary of markers used and their putative function or expression

\begin{tabular}{ll}
\hline Marker & Reported function, expression \\
\hline CD4 & Helper T cells \\
CD8 & Cytotoxic T cells \\
CD14 & Surface protein expressed by monocytes and macrophages ${ }^{49}$ \\
CD40 & Tumor necrosis receptor family, costimulatory molecule \\
CD86 & Costimulatory molecule of antigen-presenting cells \\
CD123 & IL-3 receptor $\alpha$ chain, primarily expressed on plasmacytoid dendritic cells ${ }^{50}$ \\
HLA-DR & Major histocompatibility class II antigen \\
LYVE & Hyaluronan uptake, present on luminal and abluminal surfaces of lymphatic vessels ${ }^{52}$ \\
IL-10 & Immunoregulatory cytokine produced by Th2 cells ${ }^{53}$ \\
VEGFC & Endothelial cell proliferation ${ }^{54}$, angio- and lymph- angiogenesis ${ }^{55}$ \\
VEGFR3 (also known as FLT4) & VEGF-C and -D receptors on lymphatic endothelium ${ }^{30}$ \\
Foxp3 & Immunologic self-tolerance, immune response suppression ${ }^{56}$ \\
T-bet & Transcription factor that promotes Th1 lineage commitment ${ }^{57}$ \\
GATA-3 & Transcription factor that promotes Th2 lineage commitment ${ }^{57}$ \\
Galectin 3 & Melanoma cell plasticity and vasculogenic mimicry \\
Placental growth factor & Neoangiogenesis and lymphangiogenesis \\
Osteopontin & Chemotaxis (macrophages, T cells, DCs), increased metastatic potential ${ }^{43}$ \\
HLA-G & Suppression of natural killer cell and cytotoxic T-cell cytolytic killing ${ }^{59}$ \\
\hline
\end{tabular}

were incubated overnight with antibody. Peroxidase activity was localized for all samples with $3,3^{\prime}$ diaminobenzidine and were counterstained with hematoxylin and mounted. Immunohistochemistry was performed by Histoserv (Germantown, MD, USA).

\section{Image Analysis}

We analyzed one section for each stain per patient. Owing to exhaustion of the tissue block, there were two fewer samples of sentinel lymph nodes with metastasis available for analysis of the exploratory stains (osteopontin, HLA-G, PGF, and galectin 3). Each slide was automatically scanned at $\times 20$ using $4096 \times 64$ pixel charged-couple device sensor continuous scan technology (NanoZoomer 2.0; Olympus America, Center Valley, PA, USA) to produce a virtual slide of each section. Digitized images of control tissues were examined to standardize thresholds of each stain. Standardized thresholds were then applied to determine the positively stained area of each section for each stain using IHCScore (Bacus Laboratories, Lombard, IL, USA, now Olympus America). For sentinel lymph nodes with metastasis, the areas of metastasis were not included in the analysis to exclude incorporation of expression of markers by tumor.

\section{Statistics}

Basic statistics were used to determine the median and lower and upper quartiles of each stain per group. The expression of each marker was compared between the groups using the Kruskal-Wallis test, and multiple pairwise comparisons were made using Tukey's multiple comparison test if the Kruskal-Wallis test was significant. SAS 9.1 (SAS
Institute, Cary, NC, USA) and GraphPad Prism (GraphPad Software, La Jolla, CA, USA) were used for statistical analyses and data figure preparation, respectively. A Bonferroni correction was applied because of the multiple comparisons, and a $P$-value of $\leq 0.004$ was considered significant.

\section{Results}

\section{T-Cell Polarity}

The biomarkers of T-cell polarity were primarily distributed in the $\mathrm{T}$ cell-rich paracortex of lymph nodes (Figure 1). There were significant differences in the areas of expression of the Th1 marker T-bet $(P=0.0004)$, the Th2 marker GATA-3 $(P<0.0001)$, and cytotoxic T-cell marker CD8 $(P<0.0001)$ between groups of lymph nodes. No differences were noted in the areas of expression of Foxp3 or CD4 between the groups. There were no differences between sentinel lymph nodes with or without metastasis in areas of expression of any of the T-cell markers. There was greater expression of T-bet in dormant lymph nodes (median $22.1 \%$, lower and upper quartiles $19.7-33.9 \%$ ) than reactive lymph nodes $(6.7 \%, 4.5-13.4 \%)$, sentinel lymph nodes without metastasis $(6.7 \%, 6.0-9.6 \%)$, and sentinel lymph nodes with metastasis $(4.0 \%, 3.3-12.6 \%$; all comparisons $P<0.0001)$. There was greater expression of CD8 ${ }^{+}$cells in dormant lymph nodes $(35.7 \%$, $26.0-48.2 \%)$ than reactive lymph nodes $(21.0,15.5-$ $30.2 \% ; P=0.0091)$, sentinel lymph nodes without metastasis $(1.8 \%, 1.0-2.1 \% ; P<0.0001)$ and sentinel lymph nodes with metastasis $(1.1 \%, 0.3-2.0 \%$; $P<0.0001)$ and between reactive lymph nodes, sentinel lymph nodes without metastasis $(P<0.0001)$, and sentinel lymph nodes with metastasis $(P<0.0001)$. There was greater GATA-3 expression in reactive lymph nodes $(6.4 \%, 4.5-10.6 \%)$ 


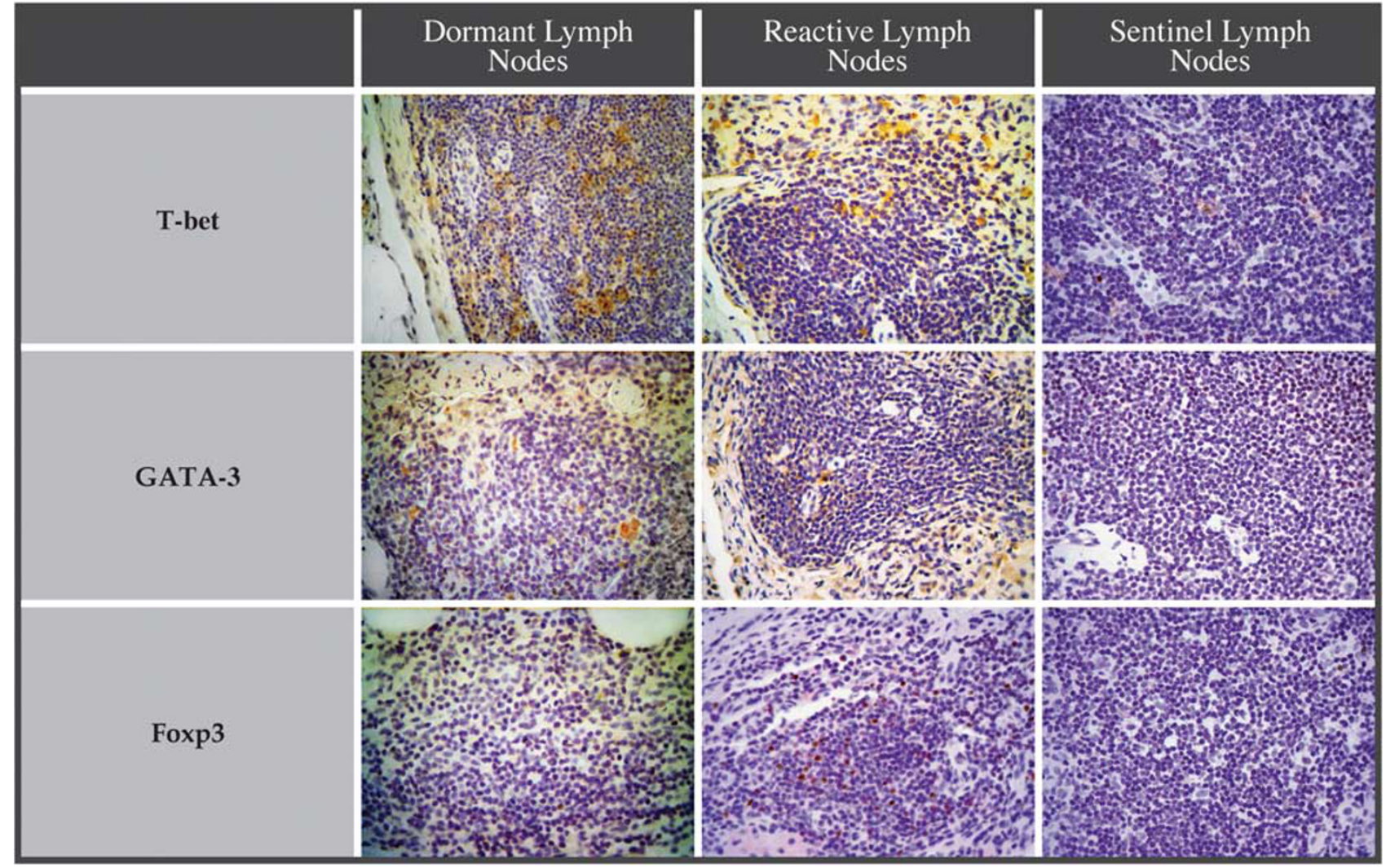

Figure 1 T-cell polarity images. Representative images at high power are shown for dormant, reactive, and sentinel lymph nodes stained for the markers of T-cell polarity, T-bet, GATA-3, and Foxp3.

than dormant lymph nodes $(0.4 \%, 0.3-0.6 \%)$, sentinel lymph nodes without metastasis $(1.9 \%$, 1.0-4.1\%), and sentinel lymph nodes with metastasis $(1.7 \%, 0.5-2.9 \%$; all comparisons $P<0.0001)$.

\section{Antigen-Presenting Cell and Costimulatory Markers}

There were significant differences in the areas of expression of CD14 $(P=0.0001)$, CD40 $(P<0.0001)$, and CD86 $(P<0.0017)$ between groups of lymph nodes. There were no differences in the areas of expression of CD123 or HLA-DR between the groups. There were no differences between sentinel lymph nodes with or without metastasis in areas of expression of any of the antigen-presenting cell and costimulatory markers. There was greater expression of CD14 in sentinel lymph nodes without metastasis $(0.94 \%, 0.49-2.27 \%)$ compared with reactive lymph nodes $(0.05 \%, 0.04-0.20 \% ; P=0.0038)$. There was greater expression of CD40 in sentinel lymph nodes without metastasis $(59.2 \%, 50.9-62.0 \%)$ than dormant lymph nodes $(32.3 \%, 20.5-49.7 \% ; P=0.0002)$ and reactive lymph nodes $(15.5 \%, 13.1-26.6 \%$; $P<0.0001)$. There was greater expression of CD40 in sentinel lymph nodes with metastasis $(61.3 \%$, $42.6-67.9 \%)$ than dormant lymph nodes $(32.3 \%$, $20.5-49.7 \% ; P<0.0001)$ and reactive lymph nodes
(15.5\%, 13.1-26.6\%; $P<0.0001)$. There was greater expression of CD86 in dormant lymph nodes $(23.8 \%, 18.3-34.7 \%)$ than reactive lymph nodes (8.6\%, 4.3-16.1\%, $P=0.0007)$, sentinel lymph nodes without metastasis $(5.2 \%, 3.6-10.5 \%$; $P<0.0001$ ), and sentinel lymph nodes with metastasis $(5.0 \%, 1.5-11.2 \% ; P<0.0001)$.

\section{Vascular Markers}

There were significant differences in the areas of expression of VEGFC $(P=0.0001)$, VEGFR3 $(P=0.0025)$, but not LYVE $(P=0.32)$, between groups of lymph nodes. There were no differences between sentinel lymph nodes with or without metastasis in areas of expression of any of the vascular markers. The greater area of expression of VEGFC in dormant lymph nodes compared with sentinel lymph nodes without metastasis was not statistically significant by pairwise comparison because of the correction factor. There was greater expression of VEGFR3 in dormant lymph nodes $(36.7 \%, 20.9-50.5 \%)$ compared with reactive lymph nodes $(7.5 \%, 6.0-12.9 \%$; $P<0.0001)$, sentinel lymph nodes without metastasis $(12.2 \%$, $8.1-18.4 \% ; P=0.0003)$, and sentinel lymph nodes with metastasis $(10.3 \%, 4.7-19.5 \% ; P=0.0005)$. 


\section{Exploratory Biomarkers of Inflammation}

There were significant differences in the areas of expression of galectin $3(P=0.0011)$, osteopontin $(P=0.0004)$ IL-10 $(P=0.0013)$, HLA-G $(P=0.0038)$, and PGF $(P=0.0014)$ between groups of lymph nodes. There were no differences between sentinel lymph nodes with or without metastasis in areas of expression of any of the exploratory biomarkers of inflammation. The differences in expression of IL10, HLA-G, and PGF were not significant by pairwise comparison. There was greater expression of galectin 3 in dormant lymph nodes $(12.5 \%$, 8.5-22.4\%) compared with sentinel lymph nodes without metastasis $(4.0 \%, 3.4-6.3 \% ; P=0.0010)$ and sentinel lymph nodes with metastasis $(2.1 \%$, $1.8-5.2 \% ; P=0.0011)$. There was greater expression of osteopontin in dormant lymph nodes $(35.1 \%$, $27.2-39.9 \%)$ compared with reactive lymph nodes (5.9\%, 3.3-22.2\%; $P<0.0001$ ), sentinel lymph nodes without metastasis (13.2\%, 6.7-15.2\%; $P<0.0001$ ), and sentinel lymph nodes with metastasis $(6.3 \%, 1.6-11.0 \% ; P<0.0001)$.

\section{Discussion}

We evaluated T-cell markers in sentinel lymph nodes and found that: (1) there were no differences between sentinel lymph nodes with or without metastasis in any of the T-cell markers we investigated, (2) dormant lymph nodes showed greater Th1 polarization than sentinel lymph nodes, and (3) there were fewer CD8 cells in sentinel lymph nodes compared with dormant and reactive lymph nodes. These results suggest that evidence of immune suppression in sentinel lymph nodes of melanoma patients is present even before metastasis. We are the first, to our knowledge, to report these findings in T-cell polarity in sentinel lymph nodes of melanoma patients. An earlier investigation, before the advent of sentinel lymph node biopsy, reported that in patients with clinical stage II disease there was no difference in T-cell markers between lymph nodes with and without metastasis. ${ }^{6}$ Although we did not look at T-cell receptors, one group found decreased expression of T-cell receptor $\zeta$ among $\mathrm{CD}^{+} \mathrm{T}$ cells in melanoma lymph nodes with metastasis compared with those without metastasis, which was thought to suggest that metastatic lymph nodes are immunosuppressed. ${ }^{7}$ We did not see any differences in $\mathrm{CD}^{+}{ }^{+}$cells between any of the lymph nodes; however, in breast cancer patients, sentinel lymph nodes without metastasis were found to have higher populations of $\mathrm{CD}^{+}$cells than sentinel lymph nodes with metastasis. ${ }^{8}$ Similar to another group, ${ }^{9}$ we also did not detect any differences in Foxp ${ }^{+}$cells between sentinel lymph nodes groups. Unlike our findings, two other groups have reported increases in $\mathrm{Foxp}^{+}$cells in melanoma-involved lymph nodes compared with those without metastasis, ${ }^{10,11}$ which is similar to results reported in breast cancer. ${ }^{12-14}$ As we did not include metastatic areas of lymph nodes in our quantification of positively stained cells in order to exclude the inclusion of positively stained melanoma cells, we may not have detected the difference noted by the other groups. Also, the lymph nodes from patients with melanoma evaluated in the other studies were from patients with palpable lymph node disease (per personal communication with authors ${ }^{10,11}$ ). Accordingly, their patient populations may represent those with more advance disease than our patients who did not have clinically detectable lymph node disease before sentinel lymph node biopsy. As circulating melanoma antigen-specific regulatory $\mathrm{T}$ cells have been reported in patients with metastatic disease, ${ }^{15}$ regulatory $\mathrm{T}$ cells may have a role in later stage disease in melanoma.

We also looked at markers of antigen-presenting cells and costimulation and found that: (1) there were no differences between sentinel lymph nodes with or without metastasis in any of the antigenpresenting cell and costimulatory markers we investigated, (2) sentinel lymph nodes demonstrated greater expression of the costimulatory marker CD40 than dormant and reactive lymph nodes, and (3) sentinel lymph nodes had diminished expression of CD86 compared with dormant and reactive lymph nodes. Although there was increased expression of costimulatory CD40 in sentinel lymph nodes, the significance of this finding is uncertain. CD40 is expressed on B cells and antigen-presenting cells, and when activated by CD40 ligand, CD40 stimulates multiple signaling pathways via tumor necrosis factor receptor-associated factors. These downstream effects are critical to the development of cellular and humoral immune responses; ${ }^{16}$ however, sole CD40 stimulation in murine melanoma vaccine studies has impaired development of durable tumor responses. ${ }^{17}$ Our findings with CD86 are similar to others showing diminished expression of this marker in peripheral blood monocytes of patients with melanoma. ${ }^{18}$ Diminished expression of CD40 and CD86 has been found in sentinel lymph nodes without metastasis when compared with nonsentinel lymph nodes without metastasis in melanoma $^{19}$ and breast cancer. ${ }^{14}$ In breast cancer, no difference in another costimulatory marker, CD83, was found between sentinel lymph nodes with or without metastasis, ${ }^{20}$ but diminished expression of CD83 has been described in sentinel lymph nodes without metastasis compared with non-sentinel lymph nodes without metastasis. ${ }^{14}$ Other groups have looked specifically at dendritic cells. Fewer interdigitating (morphologically mature) dendritic cells have been found in sentinel lymph nodes than non-sentinel lymph nodes in melanoma, ${ }^{21}$ and greater numbers of plasmacytoid dendritic cells have been found in sentinel lymph nodes with metastasis than sentinel lymph nodes without metastasis. ${ }^{22}$ Many dendritic cells in lymph nodes of melanoma patients have been found to express 
indolamine 2,3-dioxygenase. ${ }^{23}$ Indolamine 2,3-dioxygenase has been shown to mediate immunosuppression by blocking T-cell proliferation. ${ }^{24}$ Mature dendritic cells (often measured by DC-Lamp expression) have been associated with expansion of tumor antigen-specific memory effector cytotoxic T cells. Furthermore, the presence of mature dendritic cells in sentinel lymph nodes in melanoma patients is associated with absence of downstream lymph nodes metastases ${ }^{25}$ which is similar to what was found in breast cancer. ${ }^{26}$

We looked at markers of angio- and lymphangiogenesis and found that: (1) there were no differences between sentinel lymph nodes with or without metastasis in any of the markers we investigated, and (2) there was greater expression of VEGFR-3 in dormant lymph nodes compared with sentinel lymph nodes. Angiogenesis is critical for new blood vessel formation and tumor growth. ${ }^{27}$ Lymphangiogenesis is important for the metastasis of tumors. ${ }^{28}$ Both angiogenesis and lymphangiogenesis are partially controlled by VEGFs and their receptors. ${ }^{28,29}$ We decided to look at VEGF-C and VEGFR-3 expression in sentinel lymph nodes of melanoma patients. Despite many associations of VEGFR3mediated lymphangiogenesis with metastasis, ${ }^{30}$ we found greater expression of VEGFR-3 in dormant lymph nodes compared with sentinel lymph nodes. The presence of a receptor however does not imply its activation. VEGF-A and -C have been shown to promote lymphangiogenesis and metastasis in murine models of skin cancer, ${ }^{31,32}$ and in human breast cancer. ${ }^{33}$ Similarly, VEGF-D in the presence of VEGFR-3 has been associated with peritumoral lymphatic vessels and lymph node metastasis in breast cancer. ${ }^{34}$ Tumor lymphangiogenesis is predictive of metastasis in melanoma ${ }^{35-37}$ and breast cancer. ${ }^{38}$ VEGF may also have a role in immune suppression. VEGF-C has been shown not only to induce angio- and lymphangio-genesis, but it also attracts macrophages, ${ }^{39}$ which are also associated with sentinel lymph node metastasis. ${ }^{40}$ Tumorassociated macrophages demonstrate M2 polarization and promote tumor progression. ${ }^{41}$ In a previous study, our group showed that patients with stage 4 melanoma are in a systemic state of chronic inflammation (Th2 helper T-cell bias) that is likely mediated by VEGF-A. ${ }^{5}$

We looked at a number of exploratory markers and found: (1) no differences between sentinel lymph nodes with or without metastasis in any of the markers we investigated, (2) greater expression of osteopontin in dormant lymph nodes than sentinel lymph nodes and reactive lymph nodes, and (3) greater expression of galectin 3 in dormant lymph nodes than sentinel lymph nodes and reactive lymph nodes. There were no differences in the expression of IL-10, HLA-G, or PGF. Our group has taken interest in how a placenta invades the uterus and a semiallogeneic fetus escapes rejection from the maternal immune system. Accordingly, we selected a number of exploratory markers found to be important for proliferation, invasion, and immune tolerance at the fetomaternal interface. ${ }^{42}$ One of these markers, osteopontin, has chemotactic properties for macrophages, $\mathrm{T}$ cells, and dendritic cells, and is associated with increased metastatic potential. ${ }^{43}$ Regardless, we found greater expression of osteopontin in dormant lymph nodes than sentinel lymph nodes. Similar to our results, no differences in the expression of osteopontin was demonstrated between sentinel lymph nodes with or without metastasis in breast cancer. ${ }^{44}$ As osteopontin has chemotactic properties, the diminished expression of osteopontin in sentinel lymph nodes is consistent with the diminished areas of $\mathrm{CD} 8^{+} \mathrm{T}$ cells and $\mathrm{CD}^{+}{ }^{+}$cells that we observed in sentinel lymph nodes. Although galectin 3 is associated with vasculogenic mimicry and metastatic potential, ${ }^{45}$ we found diminished galectin 3 in sentinel lymph nodes without metastasis compared with dormant lymph nodes. Elevated levels of galectin 3 in serum have prognostic value in patients with regionally advanced and metastatic melanoma. ${ }^{46}$ We found a significant difference in IL-10 expression between the lymph node groups; however, there were no significant differences on pairwise comparisons, likely because of the wide variability in our data. Similar to our findings, other groups have not found any difference in IL-10 expression between sentinel lymph nodes with or without metastasis in melanoma, ${ }^{9,47}$ and disparate findings have been reported in breast cancer. ${ }^{14,20}$

This study raises the issue as to what represents an appropriate control lymph node in studies of regional lymph nodal immunity. We used both reactive and dormant lymph nodes. Whereas the dormant lymph nodes showed marked Th1 polarity, the reactive lymph nodes showed Th2 polarity. As the dormant lymph nodes were removed from patients without any known ipsilateral inflammatory process, they likely represent a resting, Th1-dominant state. On the other hand, reactive lymph nodes were excised from patients with lymphadenopathy or a concurrent inflammatory process, and likely represent the resolution of an immune response with Th2 dominance. The comparison of lymph nodes with just one of these control groups could lead to inappropriate conclusions on immune status.

In aggregate, these findings suggest that changes in regional immunity in sentinel lymph nodes precede metastasis, and may be secondary to the elaboration of soluble mediators by the primary tumor. The change in T-cell polarity, diminished CD8 cell population, and decrease in a costimulatory marker suggest that these mediators are immunosuppressive. These immunologic changes may explain the successful metastasis of melanoma in the midst of the immune environment of the sentinel lymph nodes, and lend insights into the mechanisms of lymphatic metastases in other solid malignancies. 


\section{Acknowledgements}

We thank Eric Hostetter for his assistance in the preparation of Figure 1. ASM and SGH are Outrun the Sun Research Scholars.

\section{Disclosure/conflict of interest}

The authors declare no conflict of interest.

\section{References}

1 Coit DG, Andtbacka R, Bichakjian CK, et al. Melanoma. J Natl Compr Canc Netw 2009;7:250-275.

2 Cochran AJ, Huang RR, Lee J, et al. Tumour-induced immune modulation of sentinel lymph nodes. Nat Rev Immunol 2006;6:659-670.

3 Amersi F, Morton DL. The role of sentinel lymph node biopsy in the management of melanoma. Adv Surg 2007;41:241-256.

4 Meier F, Will S, Ellwanger U, et al. Metastatic pathways and time courses in the orderly progression of cutaneous melanoma. Br J Dermatol 2002;147:62-70.

5 Nevala WK, Vachon CM, Leontovich AA, et al. Evidence of systemic Th2-driven chronic inflammation in patients with metastatic melanoma. Clin Cancer Res 2009;15:1931-1939.

6 Farzad Z, Cochran AJ, McBride WH, et al. Lymphocyte subset alterations in nodes regional to human melanoma. Cancer Res 1990;50:3585-3588.

7 Negin B, Panka D, Wang W, et al. Effect of melanoma on immune function in the regional lymph node basin. Clin Cancer Res 2008;14:654-659.

8 Kohrt HE, Nouri N, Nowels K, et al. Profile of immune cells in axillary lymph nodes predicts disease-free survival in breast cancer. PLoS Med 2005;2:e284.

9 Polak ME, Borthwick NJ, Gabriel FG, et al. Mechanisms of local immunosuppression in cutaneous melanoma. Br J Cancer 2007;96:1879-1887.

10 Jandus C, Bioley G, Speiser DE, et al. Selective accumulation of differentiated FOXP3(+) CD4 (+) T cells in metastatic tumor lesions from melanoma patients compared to peripheral blood. Cancer Immunol Immunother 2008;57:1795-1805.

11 Viguier M, Lemaitre F, Verola O, et al. Foxp3 expressing CD4+CD25(high) regulatory $\mathrm{T}$ cells are overrepresented in human metastatic melanoma lymph nodes and inhibit the function of infiltrating T cells. J Immunol 2004;173:1444-1453.

12 Mansfield AS, Heikkila PS, Vaara AT, et al. Simultaneous Foxp3 and IDO expression is associated with sentinel lymph node metastases in breast cancer. BMC Cancer 2009;9:231.

13 Matsuura K, Yamaguchi Y, Osaki A, et al. FOXP3 expression of micrometastasis-positive sentinel nodes in breast cancer patients. Oncol Rep 2009;22: 1181-1187.

14 Matsuura K, Yamaguchi Y, Ueno H, et al. Maturation of dendritic cells and T-cell responses in sentinel lymph nodes from patients with breast carcinoma. Cancer 2006;106:1227-1236.

15 Vence L, Palucka AK, Fay JW, et al. Circulating tumor antigen-specific regulatory $\mathrm{T}$ cells in patients with metastatic melanoma. Proc Natl Acad Sci USA 2007;104:20884-20889.

16 Elgueta R, Benson MJ, de Vries VC, et al. Molecular mechanism and function of CD40/CD40L engagement in the immune system. Immunol Rev 2009;229: 152-172.

17 Berner V, Liu H, Zhou Q, et al. IFN-gamma mediates CD4+ T-cell loss and impairs secondary antitumor responses after successful initial immunotherapy. Nat Med 2007;13:354-360.

18 Ugurel S, Uhlig D, Pfohler C, et al. Down-regulation of HLA class II and costimulatory CD86/B7-2 on circulating monocytes from melanoma patients. Cancer Immunol Immunother 2004;53:551-559.

19 Essner R, Kojima M. Dendritic cell function in sentinel nodes. Oncology (Williston Park) 2002;16:27-31.

20 Poindexter NJ, Sahin A, Hunt KK, et al. Analysis of dendritic cells in tumor-free and tumor-containing sentinel lymph nodes from patients with breast cancer. Breast Cancer Res 2004;6:R408-R415.

21 Cochran AJ, Morton DL, Stern S, et al. Sentinel lymph nodes show profound downregulation of antigenpresenting cells of the paracortex: implications for tumor biology and treatment. Mod Pathol 2001;14: 604-608.

22 Gerlini G, Urso C, Mariotti G, et al. Plasmacytoid dendritic cells represent a major dendritic cell subset in sentinel lymph nodes of melanoma patients and accumulate in metastatic nodes. Clin Immunol 2007; 125:184-193.

23 Lee JR, Dalton RR, Messina JL, et al. Pattern of recruitment of immunoregulatory antigen-presenting cells in malignant melanoma. Lab Invest 2003;83: 1457-1466.

24 Uyttenhove C, Pilotte L, Theate I, et al. Evidence for a tumoral immune resistance mechanism based on tryptophan degradation by indoleamine 2,3-dioxygenase. Nat Med 2003;9:1269-1274.

25 Movassagh M, Spatz A, Davoust J, et al. Selective accumulation of mature DC-Lamp+ dendritic cells in tumor sites is associated with efficient T-cell-mediated antitumor response and control of metastatic dissemination in melanoma. Cancer Res 2004;64:2192-2198.

26 Bembenek A, Li J, Loddenkemper C, et al. Presence of mature DC-Lamp+ dendritic cells in sentinel and nonsentinel lymph nodes of breast cancer patients. Eur J Surg Oncol 2008;34:514-518.

27 Folkman J. The role of angiogenesis in tumor growth. Semin Cancer Biol 1992;3:65-71.

28 Stacker SA, Achen MG, Jussila L, et al. Lymphangiogenesis and cancer metastasis. Nat Rev Cancer 2002;2: 573-583.

29 Mahabeleshwar GH, Byzova TV. Angiogenesis in melanoma. Semin Oncol 2007;34:555-565.

30 Achen MG, Stacker SA. Tumor lymphangiogenesis and metastatic spread-new players begin to emerge. Int J Cancer 2006;119:1755-1760.

31 Hirakawa S, Brown LF, Kodama S, et al. VEGF-Cinduced lymphangiogenesis in sentinel lymph nodes promotes tumor metastasis to distant sites. Blood 2007;109:1010-1017

32 Hirakawa S, Kodama S, Kunstfeld R, et al. VEGF-A induces tumor and sentinel lymph node lymphangiogenesis and promotes lymphatic metastasis. J Exp Med 2005;201:1089-1099.

33 Mohammed RA, Green A, El-Shikh S, et al. Prognostic significance of vascular endothelial cell growth 
factors -A, -C and -D in breast cancer and their relationship with angio- and lymphangiogenesis. Br J Cancer 2007;96:1092-1100.

34 van Iterson $\mathrm{V}$, Leidenius $\mathrm{M}$, von Smitten $\mathrm{K}$, et al. VEGF-D in association with VEGFR-3 promotes nodal metastasis in human invasive lobular breast cancer. Am J Clin Pathol 2007;128:759-766.

35 Dadras SS, Lange-Asschenfeldt B, Velasco $\mathrm{P}$, et al. Tumor lymphangiogenesis predicts melanoma metastasis to sentinel lymph nodes. Mod Pathol 2005;18: 1232-1242.

36 Dadras SS, Paul T, Bertoncini J, et al. Tumor lymphangiogenesis: a novel prognostic indicator for cutaneous melanoma metastasis and survival. Am J Pathol 2003;162:1951-1960.

37 Massi D, Puig S, Franchi A, et al. Tumour lymphangiogenesis is a possible predictor of sentinel lymph node status in cutaneous melanoma: a case-control study. J Clin Pathol 2006;59:166-173.

38 Skobe M, Hawighorst T, Jackson DG, et al. Induction of tumor lymphangiogenesis by VEGF-C promotes breast cancer metastasis. Nat Med 2001;7:192-198.

39 Skobe M, Hamberg LM, Hawighorst T, et al. Concurrent induction of lymphangiogenesis, angiogenesis, and macrophage recruitment by vascular endothelial growth factor-C in melanoma. Am J Pathol 2001;159: 893-903.

40 Boone B, Blokx W, De Bacquer D, et al. The role of VEGF-C staining in predicting regional metastasis in melanoma. Virchows Arch 2008;453:257-265.

41 Sica A, Schioppa T, Mantovani A, et al. Tumourassociated macrophages are a distinct M2 polarised population promoting tumour progression: potential targets of anti-cancer therapy. Eur J Cancer 2006;42: 717-727.

42 Holtan SG, Creedon DJ, Haluska P, et al. Cancer and pregnancy: parallels in growth, invasion, and immune modulation and implications for cancer therapeutic agents. Mayo Clin Proc 2009;84:985-1000.

43 Weber GF. Molecular mechanisms of metastasis. Cancer Lett 2008;270:181-190.

44 Allan AL, George R, Vantyghem SA, et al. Role of the integrin-binding protein osteopontin in lymphatic metastasis of breast cancer. Am J Pathol 2006;169: 233-246.

45 Mourad-Zeidan AA, Melnikova VO, Wang $\mathrm{H}$, et al. Expression profiling of Galectin-3-depleted melanoma cells reveals its major role in melanoma cell plasticity and vasculogenic mimicry. Am J Pathol 2008;173: 1839-1852.
46 Vereecken P, Awada A, Suciu S, et al. Evaluation of the prognostic significance of serum galectin-3 in American Joint Committee on Cancer stage III and stage IV melanoma patients. Melanoma Res 2009;19:316-320.

47 Botella-Estrada R, Dasi F, Ramos D, et al. Cytokine expression and dendritic cell density in melanoma sentinel nodes. Melanoma Res 2005;15:99-106.

48 Balch CM, Gershenwald JE, Soong SJ, et al. Final version of 2009 AJCC melanoma staging and classification. J Clin Oncol 2009;27:6199-6206.

49 Nagorsen D, Fetsch PA, Abati A, et al. Degree of CD14 expression in melanoma infiltrating mononuclear phagocytes. J Dermatol Sci 2005;37:52-54.

50 Shi J, Ikeda K, Maeda Y, et al. Identification of CD123+ myeloid dendritic cells as an early-stage immature subset with strong tumoristatic potential. Cancer Lett 2008;270:19-29.

51 Lotem M, Zhao Y, Riley J, et al. Presentation of tumor antigens by dendritic cells genetically modified with viral and nonviral vectors. J Immunother 2006;29: 616-627.

52 Banerji S, Ni J, Wang SX, et al. LYVE-1, a new homologue of the CD44 glycoprotein, is a lymphspecific receptor for hyaluronan. J Cell Biol 1999;144: 789-801.

53 Kang KH, Im SH. Differential regulation of the IL-10 gene in Th1 and Th2 T cells. Ann NY Acad Sci 2005; 1050:97-107.

54 Joukov V, Pajusola K, Kaipainen A, et al. A novel vascular endothelial growth factor, VEGF-C, is a ligand for the Flt4 (VEGFR-3) and KDR (VEGFR-2) receptor tyrosine kinases. EMBOJ 1996;15:1751.

55 Oh SJ, Jeltsch MM, Birkenhager R, et al. VEGF and VEGF-C: specific induction of angiogenesis and lymphangiogenesis in the differentiated avian chorioallantoic membrane. Dev Biol 1997;188:96-109.

56 Sakaguchi S. Naturally arising Foxp3-expressing CD25+CD4+ regulatory $\mathrm{T}$ cells in immunological tolerance to self and non-self. Nat Immunol 2005;6:345-352.

57 Jenner RG, Townsend MJ, Jackson I, et al. The transcription factors T-bet and GATA-3 control alternative pathways of T-cell differentiation through a shared set of target genes. Proc Natl Acad Sci USA 2009;106:17876-17881.

58 Loges S, Roncal C, Carmeliet P. Development of targeted angiogenic medicine. J Thromb Haemost 2009;7:21-33.

59 Hunt JS, Langat DK, McIntire RH, et al. The role of HLA-G in human pregnancy. Reprod Biol Endocrinol 2006;4(Suppl 1):S10. 